\title{
Increasing HIV testing engagement through provision of home HIV self-testing kits for patients who decline testing in the emergency department: a pilot randomisation study
}

\author{
Anuj V Patel, ${ }^{1}$ Samuel M Abrams, ${ }^{1}$ Charlotte A Gaydos, ${ }^{1,2}$ Mary Jett-Goheen, ${ }^{2}$ \\ Carl A Latkin, ${ }^{3}$ Richard E Rothman, ${ }^{1,2}$ Yu-Hsiang Hsieh ${ }^{1}$
}

'Department of Emergency Medicine, The Johns Hopkins University School of Medicine, Baltimore, Maryland, USA ${ }^{2}$ Department of Medicine, Division of Infectious Diseases, School of Medicine, Johns Hopkins University, Baltimore, Maryland, USA

${ }^{3}$ Department of Health, Behavior and Society, Bloomberg School of Public Health, Johns Hopkins School of Public Health, Baltimore, Maryland, USA

\section{Correspondence to} Professor Yu-Hsiang Hsieh, Department of Emergency Medicine, The Johns Hopkins University School of Medicine, Baltimore, MD 21205, USA; yhsieh1@jhmi.edu

AVP and SMA contributed equally.

Part of this work was presented at the 2016 Society of Academic Emergency Medicine Midwest Regional Meeting, 8-9 September 2016, Indianapolis, Indiana, USA, and at the 2017 Society of Academic Emergency Medicine Annual Meeting, 16-19 May 2017, Orlando, Florida, USA.

Received 26 February 2018 Revised 24 April 2018

Accepted 20 May 2018 Published Online First 14 June 2018

\section{ABSTRACT}

Objective Up to $60 \%$ of patients decline routine HIV testing offer in US emergency departments (EDS). The objective of this study is to determine whether the provision of HIV self-testing (HIVST) kit would increase engagement of HIV testing among these HIV test 'Decliners'.

Methods Patients who declined a test offered in an ED-based triage nurse-driven HIV screening programme were enrolled and randomised to either the HIVST or the control group. The patients in the HIVST group received HIVST kits to take home, were encouraged to report test results to an established internet-based STI/HIV testing recruitment website 'I Want the Kit' (IWTK) and received five referral cards for their peers to request HIVST kits from IWTK. The control group received pamphlets about publicly available HIV testing sites. HIV testing from both groups after enrolment was determined via telephone follow-up at 1 month. Testing rate ratio (RR) was determined using $\chi^{2}$ tests.

Results Fifty-two patients were randomised to the HIVST group and 48 to the control group. Among all 64 patients completing any follow-up, 14/29 (48\%) patients in the HIVST group tested themselves at home with the provided kit. Four of these had never had an HIV test. Only 2/35 (6\%) in the control group reported having an HIV test after enrolment (RR: 8.45 (95\% Cl: 2.09 to $34.17)) .57 \%(8 / 14)$ in the HIVST group reported test results to IWTK.

Conclusion Provision of HIVST kits supplements EDbased screening programme and significantly improved engagement of HIV testing among those test 'Decliners' in the ED.

Trial registration number NCT03021005, results.

\section{INTRODUCTION}

Emergency departments (EDs) have identified thousands of undiagnosed patients and linked the majority of them to HIV care. ${ }^{1}$ Despite efforts to streamline the testing programme, up to $60 \%$ of the patients decline HIV testing from a non-targeted opt-out screening programme in urban EDs. ${ }^{2}$ Novel testing strategies and approaches to overcome factors associated with HIV test decline in EDs have been proposed. ${ }^{34}$ One of such approaches that have been demonstrated to be feasible and acceptable is HIV self-testing (HIVST). ${ }^{5}$

Our objective was to offer home testing to those patients who declined HIV testing in EDs and engage more patients in HIV testing by providing an opportunity for patients to test themselves confidentially and in private. We planned an innovative approach of providing a free HIVST kit to take home for patients who had just declined an HIV test offer in EDs, 'Decliners'. The aim of this study was to determine whether provision of HIVST kits to HIV testing 'Decliners' in EDs would increase testing engagement.

\section{METHODS}

From February to May of 2016, a randomised study was conducted in an urban ED where a routine triage nurse-driven non-targeted opt-out HIV screening programme operated. Patients aged $\geq 18$ years, who declined HIV screening during the visit and were able to provide a verbal consent, were eligible. Eligible patients on discretion of clinicians were referred to a research coordinator during weekdays between 09:00 and 17:00. Consented patients took a baseline survey on a tablet before being randomised to the HIVST or the control group. The 31 survey questions inquired about patient's sociodemographics, HIV testing history, reasons of declining testing offer at triage, willingness to take home an HIVST kit and use it. Randomisation was double-blinded based on 110 sealed envelopes prepared by a third person with 1:1 preassigned randomisation allocation.

HIVST patients received the Food and Drug Administration-approved home use oral-fluid HIVST kit, OraQuick In-Home HIV Test (OraSure, Bethlehem, Pennsylvania, USA). Instruction sheets on how to perform HIVST and interpret the test results were provided. They were encouraged to report HIVST test results with the specific unique codes provided on the instruction sheet to an established internet-based STIs/HIV testing recruitment website-'I Want the Kit (IWTK)' (www.iwantthekit.org), for recruiting participants to self-collect samples at home and mail them to a laboratory to test for STIs. Whereas patients in the control group received an informational pamphlet on the 
importance of HIV testing and list of testing sites in Baltimore. Both instruction sheet and informational pamphlet had the Flesch-Kincaid reading scale of the fifth grade level. All enrolled patients were followed up by phone 1 month after enrolment.

Follow-up phone surveys consisted of questions regarding any HIV testing after enrolment. HIVST patients were additionally asked if they used the provided HIVST kit to test themselves and reported their self-test results on the IWTK website, their perception of HIVST and whether the participant was able to distribute referral cards to partners and peers.

\section{Statistical analysis}

Descriptive data analyses were performed using SAS V.9.4. Rate ratio (RR) was used for comparison of HIV testing rates between two groups by the $\chi^{2}$ test.

\section{RESULTS}

Overall, 100 (79\%) of 126 referred patients were enrolled. Fifty-two patients were randomised to the HIVST group and 48 patients to the control group. Patients' sociodemographics are summarised in table 1. Regarding HIV testing history, 43 (43\%) patients had not been tested during last year, including 16 patients reporting that they have never been tested for HIV. The main reasons for declining an ED-based HIV testing were their perception of not being at risk $(n=40)$ and recent HIV testing $(n=39)$. There were no statistically significant differences in sociodemographics between the HIVST and control group. All HIVST participants agreed to take home an HIVST kit.

At a 1-month follow-up, 64 participants provided their HIV testing information after the enrolment. Fourteen (48\%) of 29 HIVST patients reported being tested for HIV using the HIVST kit provided, while $2(6 \%)$ of 35 patients in the control group reported being tested at the medical care setting (RR: 8.45 (95\% CI: 2.09 to 34.17$)$ ). Eight (57\%) of 14 HIVST patients who tested themselves reported their results to IWTK. Noticeably, of the 16 patients who had never been tested for HIV, 4 (40\%) of 10 HIVST participants reported that they used the provided HIVST kit to test themselves while $1(17 \%)$ of 6 patients in the control group reported receiving HIV testing after the enrolment. Among 29 patients in the HIVST group whom we were able to follow at 1 month, 10 (34\%) reported that they had distributed referral cards to their partners and peers. However, none of the referral cards were used to request for an HIVST kit from IWTK.

\section{DISCUSSION}

In this study, our findings demonstrate that the provision of HIVST kits significantly increased HIV testing rate in ED HIV testing 'Decliners'. Our approach provides an additional opportunity to engage patients who decline HIV testing, including those who had never been tested for HIV. In addition to the increased testing engagement, we noticed that HIVST patients accepted our ED-based intervention strategy, reflected by the fact that all HIVST patients took the provided kits home, many of them tested themselves at home and provided favourable comments at the phone follow-up (data not shown).

One of the main reasons for the significant increase in HIV testing engagement in HIVST patients could be that HIVST at home provides patients the comfort of testing at their own home, with complete confidentiality and privacy. Home HIVST could potentially eliminate the stigma associated with accepting an HIV test in EDs. ${ }^{6}$ The availability of supplementary HIV testing modalities such as the provision of HIVST kits might be a key
Table 1 Sociodemographics of 100 emergency department (ED) patient participants who declined HIV testing in the ED, by the intervention, provision of home HIV self-testing (HIVST) kits

\begin{tabular}{|c|c|c|c|c|}
\hline \multirow[b]{3}{*}{ Characteristics } & \multirow[b]{3}{*}{ Category } & \multirow{2}{*}{$\begin{array}{l}\text { Total } \\
\text { number } \\
(\%)\end{array}$} & \multicolumn{2}{|c|}{ Number (\%) } \\
\hline & & & $\begin{array}{l}\text { HIVST } \\
\text { group }\end{array}$ & $\begin{array}{l}\text { Control } \\
\text { group }\end{array}$ \\
\hline & & $n=100$ & $n=52$ & $\mathrm{n}=48$ \\
\hline \multirow[t]{5}{*}{ Age (years) } & $18-24$ & $10(10)$ & $6(12)$ & $4(8)$ \\
\hline & $25-34$ & $28(28)$ & $18(35)$ & $10(21)$ \\
\hline & $35-44$ & $28(28)$ & $13(25)$ & $15(31)$ \\
\hline & $45-54$ & $21(21)$ & $8(15)$ & $13(27)$ \\
\hline & $\geq 55$ & $13(13)$ & $7(13)$ & $6(13)$ \\
\hline \multirow[t]{2}{*}{ Gender } & Male & 34 (34) & $17(33)$ & $17(35)$ \\
\hline & Female & $66(66)$ & $35(67)$ & $31(65)$ \\
\hline \multirow[t]{3}{*}{ Race } & White & $46(46)$ & $26(50)$ & $20(42)$ \\
\hline & African-American & $43(43)$ & $21(40)$ & $22(45)$ \\
\hline & Other & $11(11)$ & $5(10)$ & $6(13)$ \\
\hline \multirow[t]{3}{*}{ Ethnicity } & Non-Hispanic & $84(84)$ & $40(77)$ & $44(92)$ \\
\hline & Hispanic & $4(4)$ & $4(8)$ & $0(0)$ \\
\hline & Unknown & $12(12)$ & $8(15)$ & $4(8)$ \\
\hline \multirow[t]{5}{*}{ Education } & Grade 12 or less & $16(16)$ & $8(15)$ & $8(17)$ \\
\hline & Technical/vocational & $4(4)$ & $1(2)$ & $3(6)$ \\
\hline & $\begin{array}{l}\text { High school graduate or } \\
\text { equivalent }\end{array}$ & $13(13)$ & $8(15)$ & $5(10)$ \\
\hline & Some college or higher & $22(22)$ & $9(17)$ & $13(27)$ \\
\hline & Graduate/college degree & $45(45)$ & $26(50)$ & $19(40)$ \\
\hline \multirow{5}{*}{$\begin{array}{l}\text { Sexual } \\
\text { orientation }\end{array}$} & Heterosexual & $88(88)$ & $48(92)$ & $40(83)$ \\
\hline & Bisexual & $4(4)$ & $3(6)$ & $1(2)$ \\
\hline & $\begin{array}{l}\text { Man who has sex only with } \\
\text { men }\end{array}$ & $0(0)$ & $0(0)$ & $0(0)$ \\
\hline & $\begin{array}{l}\text { Woman who has sex only } \\
\text { with women }\end{array}$ & $5(5)$ & $1(2)$ & $4(8)$ \\
\hline & Did not answer & $3(3)$ & $0(0)$ & $3(6)$ \\
\hline \multirow[t]{5}{*}{ Insurance } & None & $6(6)$ & $4(8)$ & $2(4)$ \\
\hline & Medicaid/medicare & $36(36)$ & $17(32)$ & $19(40)$ \\
\hline & Private & $51(51)$ & $29(56)$ & $2(46)$ \\
\hline & Veterans administrations & $4(4)$ & $1(2)$ & $3(6)$ \\
\hline & Do not know & $3(3)$ & $1(2)$ & $2(4)$ \\
\hline \multirow{2}{*}{$\begin{array}{l}\text { Primary care } \\
\text { doctor }\end{array}$} & Yes & $83(83)$ & $43(83)$ & $40(83)$ \\
\hline & No & $17(17)$ & $9(17)$ & $8(17)$ \\
\hline \multirow{2}{*}{$\begin{array}{l}\text { Injection drug } \\
\text { use }\end{array}$} & Current & $2(2)$ & $1(2)$ & $1(2)$ \\
\hline & In the past & $4(4)$ & $3(6)$ & $1(2)$ \\
\hline \multirow{3}{*}{$\begin{array}{l}\text { HIV testing } \\
\text { frequency in the } \\
\text { past } 12 \text { months }\end{array}$} & 0 & $43(43)$ & $22(42)$ & $21(44)$ \\
\hline & 1 & 34 (34) & $19(37)$ & $15(31)$ \\
\hline & $>2$ & $23(23)$ & $11(21)$ & $12(25)$ \\
\hline
\end{tabular}

strategy to reach testing decliners to increase their engagement for testing or even identify hidden undiagnosed infections.?

Our study is one of the pioneer studies to provide kits to testing decliners in the ED setting, and many of whom had never been tested in their lifetime. Many patients, who perceived themselves at no risk for HIV or felt uncomfortable to have the HIV test performed in EDs, declined HIV testing offer as part of routine ED services. ${ }^{289}$ Thus, it is imperative to use acceptable alternate approaches to supplement the conventional ED-based clinician-driven testing programme in order to engage patients into HIV testing.

One-third of HIVST patients reported that they distributed the referral cards to their partners and/or peers for them to request an HIVST kit from IWTK. However, none used the referral cards 
to request an HIVST kit. Several studies that applied peer-based referral approach to penetrate the social network of patients for engagement of their peers into HIV testing have had varied successes and shortcomings. ${ }^{10}$ Since this was not our primary outcome of this study, we were uncertain about the reasons why only so few participants distributed the referral cards and why there were no requests for HIVST kits from the peers. One of the likely reasons may be that two-thirds of the participants failed to distribute the referral cards, reflecting concerns and fears about how their partners or peers perceived. Alternatively, one possible reason for the lack of requests is that peers might have perceived themselves at no risk for HIV. Further studies using a mixedmethod research approach to understand how decliners and their partners and peers feel regarding the social network referral for increasing engagement of HIV testing are warranted, in order to develop a gender, racially, and culturally sensitive referral tool for this population, who perceive themselves at no risk.

Our study has several limitations. First, this was a single institution study, which could limit our findings from being generalised to other EDs. Second, our outcomes on HIV testing were based on patient self-reporting. Thus, our study is potentially susceptible to social desirability bias. Third, with a follow-up rate of $64 \%$, which is similar to studies conducted in our ED, we might have underestimated or overestimated the RR due to the loss of follow-up. Finally, with a readability level of the fifth grade for both self-testing instruction and the informational pamphlet, this might have discouraged some participants to use HIVST kit or seek for a free HIV testing site. However, we expect that literacy would not bias our outcomes towards one group of participants due to the nature of randomisation.

In summary, we demonstrated that provision of home HIVST kits to patients, who had just declined an HIV test in EDs, resulted in at least eight times more HIV testing among the 'Decliners' 1 month later compared with the control group. This approach changed the minds of patients, many of whom had never been tested for HIV and perceived themselves at no risk for HIV. We believe that this novel 'Take an HIV self-testing kit home' strategy could supplement the current ED-based HIV screening programmes in order to increase the overall HIV testing uptake.
Handling editor Jackie A Cassell

Contributors $\mathrm{Y}$-HH designed the study. SMA, CAG, MJ-G and RER provided critical suggestions to assist $\mathrm{Y}-\mathrm{HH}$ to finalise the study protocol. SMA, AVP and MJ-G had primary responsibility for the data collection. AVP and SMA conducted the phone follow-up survey. Y-HH performed data analyses. Y-HH, SMA, AVP, CAG and RER primarily interpreted results. Y-HH and AVP primarily drafted the manuscript. SMA, CAG, RER, MJ-G and CAL performed critical editing of the manuscript.

Funding CAG, MJ-G, RER and Y-HH are supported by National Institutes of Health (NIH) U54EB007958. CAG is also supported by U01-068613, National Institute of Allergy and Infectious Diseases, NIH.

Competing interests None declared.

Patient consent Not required.

Ethics approval Johns Hopkins School of Medicine Institutional Review Board.

Provenance and peer review Not commissioned; externally peer reviewed.

(c) Article author(s) (or their employer(s) unless otherwise stated in the text of the article) 2019. All rights reserved. No commercial use is permitted unless otherwise expressly granted.

\section{REFERENCES}

1 Haukoos JS. The impact of nontargeted HIV screening in emergency departments and the ongoing need for targeted strategies. Arch Intern Med 2012;172:20-2.

2 Haukoos J, Lyons M, Rothman R, et al. The HIV Tested Trial: A Multi-center Pragmatic Randomized Comparison of HIV Screening Strategy Effectiveness inthe Emergency Department. Acad Emerg Med 2017;24(S1):S42-3.

3 Hsieh YH, Gauvey-Kern M, Peterson S, et al. An emergency department registration kiosk can increase HIV screening in high risk patients. J Telemed Telecare 2014:20:454-9.

4 Aronson ID, Cleland CM, Perlman DC, et al. Feasibility of a Computer-Based Intervention Addressing Barriers to HIV Testing Among Young Patients Who Decline Tests at Triage. J Health Commun 2016;21:1039-45.

5 Gaydos CA, Hsieh YH, Harvey L, et al. Will patients "opt in" to perform their own rapid HIV test in the emergency department? Ann Emerg Med 2011;58(1 Suppl 1):S74-S78.

6 Christopoulos KA, Weiser SD, Koester KA, et al. Understanding patient acceptance and refusal of HIV testing in the emergency department. BMC Public Health 2012;12:3.

7 Hsieh YH, Kelen GD, Beck KJ, et al. Evaluation of hidden HIV infections in an urban ED with a rapid HIV screening program. Am J Emerg Med 2016;34:180-4.

8 Brown J, Kuo I, Bellows J, et al. Patient perceptions and acceptance of routine emergency department HIV testing. Public Health Rep 2008;123 Suppl 3(Suppl 3):21-6.

9 Pisculli ML, Reichmann WM, Losina E, et al. Factors associated with refusal of rapid HIV testing in an emergency department. AIDS Behav 2011;15:734-42.

10 Gwadz M, Cleland CM, Perlman DC, et al. Public Health Benefit of Peer-Referral Strategies for Detecting Undiagnosed HIV Infection Among High-Risk Heterosexuals in New York City. J Acquir Immune Defic Syndr 2017;74:499-507. 